\title{
What did we learn from South Africa's first-ever tuberculosis prevalence survey?
}

\author{
Y Pillay, ${ }^{1} \mathrm{PhD} ; \mathrm{L}$ Mvusi, ${ }^{2} \mathrm{MB}$ ChB; L D Mametja, ${ }^{3} \mathrm{MPH} ;$ S Dlamini, ${ }^{2} \mathrm{MPH}$ \\ ${ }^{1}$ Clinton Health Access Initiative, Pretoria, South Africa \\ ${ }^{2}$ National Department of Health, Pretoria, South Africa \\ ${ }^{3}$ Health Professions Council of South Africa, Pretoria, South Africa
}

Corresponding author: Y Pillay (ygpillay@gmail.com)

The World Health Organization (WHO) has urged countries to conduct tuberculosis (TB) prevalence surveys to better understand the burden of TB and to enable the WHO to conduct global estimates. Until the report from the first-ever prevalence survey in South Africa (SA), the country had to rely on WHO estimates. The recently published report on the SA TB prevalence survey provides important estimates of the burden of TB disease as well as information on health-seeking behaviour. This review notes the key findings of the 2018 prevalence survey. The high prevalence of TB in SA continues to be a major cause for concern, and calls for a significantly improved response to reach the End TB targets set by the WHO.

S Afr Med J. Published online 6 April 2021. https://doi.org/10.7196/SAMJ.2021.v111i5.15662

The first-ever tuberculosis (TB) prevalence survey in South Africa (SA) was conducted in 2018, with the results publicly announced by the Minister of Health in February 2021. It is remarkable that although SA is one of eight countries responsible for two-thirds of the world's TB cases, this was the first survey to estimate the prevalence of TB in the country. Previously, the burden of TB was estimated through modelling exercises by the World Health Organization (WHO). However, these estimates were sometimes unreliable, as they were based on unadjusted assumptions. In 2013 the WHO reported that SA's TB incidence was as high as $1000 / 100$ 000, based on 2012 case-finding data, ${ }^{[1]}$ and in 2019 the organisation reported a TB incidence of 520/100 000, based on 2018 case-finding data ${ }^{[2]}$ It was hoped that the first-ever prevalence survey would provide a more reliable estimate of SA's true TB burden.

Why are TB prevalence surveys conducted, and how are their results used? According to Colvin and Castro: ${ }^{[3]}$ 'Tuberculosis (TB) prevalence surveys provide the most accurate measure of the burden of disease and data for monitoring disease trends over time. The results of these surveys are also used to calibrate mathematical models to forecast the extent and burden of TB around the globe.'

The surveys are not only important for the individual countries in which they are being conducted to know the burden and to plan interventions, but they also generate global estimates of the prevalence of TB. Without such surveys, countries typically rely on WHO modelling to estimate their prevalence and on independent programme reviews to understand the performance of the national TB programmes. SA has conducted such independent reviews supported by the WHO, bilateral agencies such as the US Centers for Disease Control and Prevention (CDC) and US Agency for International Development (USAID) and technical partners over the past decade. These review recommendations were used to strengthen the national TB programme.

The WHO urged SA to undertake a prevalence survey in 2008, but resources could only be mobilised in 2016. With financial support from a range of donors, the National Department of Health was able to commission the Human Sciences Research Council, the South
African Medical Research Council and the National Institute of Communicable Diseases to conduct the TB prevalence survey.

\section{What's known about TB in SA as reported by the WHO's 2020 Global TB Report?}

The global TB burden is reported annually by the WHO in its Global TB Report, with detailed accounts for what are known as highburdened countries, currently 30 in number, including SA. In its 2020 Global TB Report, ${ }^{[4]}$ the WHO noted that SA is one of eight countries that contributed two-thirds of the global burden of TB disease - with SA accounting for $3.6 \%$. The report estimated that the incidence of TB in SA, based on 2019 case-finding data, was 615/100 000 (range 427 - 835), compared with 520 (range 373 - 691) reported in 2019 using 2018 case-finding data.

Of concern is that SA is one of four countries that showed large declines in TB notifications during the first 6 months of 2020, with notifications falling by $50 \%$ between March and June 2020 - the result of the COVID pandemic and severe lockdowns that make access to health facilities challenging.

The 2020 Global TB Report ${ }^{[4]}$ does contain some positive news: SA is one of the 78 countries globally that were reported to be on track to achieve a 2020 milestone of a $20 \%$ decrease in TB incidence.

SA has been among the early adopters of new anti-TB medication. In 2019, SA, India, the Russian Federation and Ukraine accounted for $68 \%$ of all patients treated with bedaquiline globally. In addition, SA, with India and Tanzania, initiated $56 \%$ of patients on TB preventive therapy globally. In terms of the extent of domestic funding for the national TB programme, the SA government provides $81 \%$ of total funding for the programme.

\section{Objectives and design of the prevalence survey}

The objectives of the TB prevalence survey were to: $(i)$ estimate the prevalence of pulmonary TB at a national level in people aged $\geq 15$ years; and (ii) identify the extent to which those with pre- 
existing TB or with symptoms of pulmonary TB seek care, and if so from which health facilities. The survey sampled individuals aged $\geq 15$ years who could provide consent from 110 clusters across all nine provinces. A sample size of 54873 was drawn using a stratified sampling design. ${ }^{[5]}$

Symptom and chest radiograph (CXR) screening was conducted on all participants and an Xpert Ultra (Cepheid) test and TB culture were conducted on all those with a positive screen. As far as we are aware, SA was one of a few countries that used Xpert Ultra in conducting a prevalence survey. The use of Xpert Ultra raised concern regarding false-positive results if used in low pre-test probability settings, which posed a challenge in defining a TB case under survey conditions. The WHO facilitated a process for standardisation of a TB case definition for prevalence surveys.

The case definition that was used is as follows:

- Any Mycobacterium tuberculosis complex culture-positive case, irrespective of Xpert Ultra results

- Negative culture for M. tuberculosis complex conditional on:

(i) positive Xpert Ultra

(ii) no history of, or current TB

(iii) findings on CXR suggestive of active TB (as confirmed by an external CXR reading panel).

\section{Survey participation and results}

Of the 53250 people eligible to participate in the survey who were sampled, 35191 participated. This means that the overall participation rate was $66.1 \%$, which was lower than the expected $85 \%$. Participation was lower for men than women, and among the youth and people living in urban areas. The lowest participation rates were for men aged 25 - 34 years and the highest rates for women aged 55 - 64 years. ${ }^{[5]}$

The data were adjusted for biases in the sampling, especially the low participation rates in urban areas as well as the high number of false positives from the Xpert Ultra tests. However, the wide confidence levels reflected in Table 1 suggest the level of uncertainty in the results. Also, the high rates found in the survey should be a concern.

The overall prevalence of TB in SA was estimated to be 737 per 100000 population (95\% confidence interval $580-890)$. The prevalence was significantly higher in males than in females. This is similar to the findings in prevalence surveys in Kenya ${ }^{[6]}$ and Vietnam. ${ }^{[7]}$ In the Vietnam survey, men were four times more likely to have TB than women. Of those surveyed, $32.1 \%$ reported no symptoms of TB, but $97.7 \%$ of those who reported no symptoms had CXR images suggestive of TB. Prevalence was estimated to be lowest in those aged 15 - 24 years and highest amongst those aged $\geq 65$ years.

The prevalence survey also reported on health-seeking behaviour among participants with TB symptoms. The majority of those who reported seeking care for their TB symptoms did so at public health clinics (90\%), with only $8.1 \%$ seeking care in the private health sector. This difference probably reflects ability to pay for services in the private sector rather than confidence in the health services offered by the public sector, given that for general health services, the same proportion uses the public sector health services. When compared with the number of survey participants who sought care in the private sector, the TB prevalence survey found that $21 \%$ of participants sought care in the private sector. ${ }^{[6]}$

Of significant concern was the finding that of 5168 participants in the survey who reported at least one symptom, 66.6\% ( $n=3442) \mathrm{did}$ not visit a health facility, even though $60.2 \%$ of them reported that they intended to seek care. Among those with symptoms who did not seek care, $26.6 \%$ reported not seeking care because they did not consider their symptoms to be serious enough. Participants who were HIV-positive were more likely to have sought care when they had symptoms compared with those who were HIV-negative.

In addition, as is the case for other health conditions, younger people with symptoms were least likely to seek care. The delay in care seeking is of concern for the following reasons, according to the WHO: ${ }^{[8]}(i)$ the possibility of transmission - each person with symptoms can infect between 5 and 15 others over a lifetime; (ii) disease progression in the individual with untreated TB; and (iii) mortality due to undiagnosed TB disease.

The proportion of survey participants who reported no symptoms but were found to have TB disease was high, at $57.8 \%$. This finding was similar to findings in other countries, which highlights a significant gap in TB detection with the current reliance on symptom screening as an entry point to testing and treatment.

\section{What the survey did not tell us}

While many new data were obtained from the first-ever national TB prevalence survey, the survey was not designed to measure the prevalence in children aged $<15$ years, or their access to health services. In addition, it was not designed to provide estimates at subnational level (either provincial- or district-level data).

According to the 2020 Global TB Report, ${ }^{[4]}$ there are five risk factors for TB. These are: (i) being HIV-positive; (ii) undernutrition; (iii) smoking, especially in men; (iv) alcohol use disorders; and ( v) diabetes. The survey provided estimates of HIV-positive persons with TB but not any of the other risk factors.

\section{What should be done with the survey resullts?}

Although the 15 - 24-year-old age group has a relatively low prevalence of TB (423/100 000), they have the largest prevalence-

Table 1. The major findings of the TB prevalence survey ${ }^{[5]}$

\begin{tabular}{lll}
\hline Category & Prevalence of TB, per 100 000 population & 95\% CI \\
\hline Female & 675 & $494-855$ \\
Male & 1094 & $835-1352$ \\
Age groups (years) & & $232-632$ \\
$\quad 15-24$ & 432 & $583-1221$ \\
$25-34$ & 902 & $703-1511$ \\
$35-44$ & 1107 & $682-1443$ \\
$45-54$ & 1063 & $505-1186$ \\
$55-64$ & 845 & $680-1528$ \\
$\quad \geq 65$ & 1104 &
\end{tabular}


to-notification gap, of 2.91. They should be prioritised for targeted intervention. Researchers have found that in India, Indonesia, the Philippines and Pakistan, 15 -19-year-olds were involved in 20\% of TB transmission even though they represented $5 \%$ of local TB incidence. ${ }^{[9]}$ Equally, diagnosing TB in the elderly is often neglected by many countries, ${ }^{[10]}$ yet people in this age group have many comorbidities and attend health facilities frequently, and should therefore be offered TB tests routinely.

New models of service delivery are required to reach men and young people as well as those with asymptomatic TB. For example, men's clinics and youth-friendly clinics, ${ }^{[11]}$ workplace clinics (for example in the mines), and intensified screening and testing in community settings are required. For asymptomatic TB, screening and testing of all TB contacts should be expanded and the use of digital hand-held portable X-ray machines should be considered.

The social determinants of TB are often neglected. SA is one of the most unequal countries in the world. The negative impact of the COVID pandemic as well as the structural weakness of the SA economy has resulted in increasing unemployment and poverty. While a social safety net exists in the form of government grants, levels of hunger are high, as found by the National Income Dynamics Survey. ${ }^{[12]}$ The results of the national patient cost survey designed to estimate the catastrophic costs incurred by TB patients and their families will provide more insight into the SA situation.

\section{Conclusions}

The prevalence of $\mathrm{TB}$ in $\mathrm{SA}$ is high and requires increased determination, effort and resources. Too many people with TB are missed, especially men, young people and those aged $>60$ years. To eliminate TB as a public health threat, SA needs to address the social, economic and health-related factors that contribute to acquisition and transmission of the disease. Involvement by government of all stakeholders, in particular families, communities and employers, will be key to mounting a response that will bring together all of government and all of society.

There are many lessons for $\mathrm{TB}$ prevention and management from the country's response to the COVID pandemic - both of which are airborne infections. We need to rapidly take the lessons from our response to COVID-19 and apply them to TB prevention and management, including agile decision-making, involving all stakeholders, using mobile technology, real-time surveillance systems, enhanced political leadership and additional resources.

\section{Declaration. None.}

Acknowledgements. None.

Author contributions. YP conceptualised and drafted the manuscript. LM, LDM and SD revised and edited the manuscript.

Funding. None.

Conflicts of interest. None.

1. World Health Organization. Global Tuberculosis Report 2013. Geneva: WHO, 2013. https://apps.who. int/iris/bitstream/handle/10665/91355/9789241564656_eng.pdf?sequence=1\&isAllowed=y (accessed 26 February 2021).

2. World Health Organization. Global Tuberculosis Report 2019. Geneva: WHO, 2019. https://apps.who. int/iris/bitstream/handle/10665/329368/9789241565714-eng.pdf (accessed 20 February 2021).

3. Colvin CE, Castro KG. Independent assessment of national TB prevalence surveys conducted between 2009 - 2015. US Agency for International Development (USAID), February 2016. https://www usaid.gov/sites/default/files/documents/1864/TB-prevalence-surveys-assessment-2016-508-final.pdf (accessed 17 February 2021)

4. World Health Organization. Global Tuberculosis Report 2020. Geneva: WHO, 2020. https://www.who. int/publications/i/item/9789240013131 (accessed 17 February 2021).

5. Van der Walt M, Moyo S. The First National TB Prevalence Survey, South Africa 2018: Short report. 2021. https://www.knowledgehub.org.za/system/files/elibdownloads/2021-02/A4_SA_TPS\%20Short\%20 Report_10June20_Final_highres.pdf (accessed 17 February 2021).

6. Enos M, Sitienei J, Ongango J, et al. Kenya tuberculosis prevalence survey 2016: Challenges and opportunities of ending TB in Kenya. PLoS ONE 2018;13(12):e0209098. https://doi.org/10.1371/ journal.pone. 0209098

7. Nguyen HV, Tiemersma EW, Nguyen HB, et al. The second national tuberculosis prevalence survey in Vietnam. PLoS ONE 2020;15(4):e0232142. https://doi.org/10.1371/journal.pone.0232142

8. World Health Organization. Tuberculosis: Key facts. 14 October 2020. https://www.who.int/newsroom/fact-sheets/detail/tuberculosis (accessed 17 February 2020).

9. Ragonnet R, Trauer JM, Geard N, et al. Profiling Mycobacterium tuberculosis transmission and the resulting disease burden in the five highest tuberculosis burden countries. BMC Med 2019;17:208. resulting disease burden in the five highes
https://doi.org/10.1186/s12916-019-1452-0

10. Kak N, Chakraborty K, Sadaphal S, et al. Strategic priorities for TB control in Bangladesh, Indonesia, and the Philippines - comparative analysis of national TB prevalence surveys. BMC Public Health 2020;20:560. https://doi.org/10.1186/s12889-020-08675-9

11. Doyle AM, Mchunu L, Koole O, et al. Primary healthcare and school health service utilisation by adolescents and young adults in KwaZulu-Natal, South Africa. BMC Health Serv Res 2019;19:905. https://doi.org/10.1186/s12913-019-4559-2

12. Van der Berg S, Patel L, Bridgman G. Hunger in South Africa during 2020: Results from Wave 3 of NIDS-CRAM. National Income Dynamics Study (NIDS)-Coronavirus Rapid Mobile Survey (CRAM) 17 February 2021 https//cramsurvey org/wp-content/uploads/2021/02/10. Van-der-Berg-S.-PateBridgman-G.-2021-Hunger-in-South-Africa-during-2020-Results-from-Wave-3-of-NIDS-CRAM-1. pdf (accessed 17 February 2021)

Accepted 8 March 2021. 